\title{
Finite-difference Time-domain Study on Birefringence Changes of the Axon During Neural Activation
}

\author{
Jonghwan Lee \\ Graduate School of Neuroscience, Seoul National University, Seoul 151-742, Korea \\ Sung June Kim* \\ School of Electrical Engineering and Computer Science, Seoul National University, Seoul 151-742, Korea
}

(Received April 15, 2009 : revised May 25, 2009 : accepted May 25, 2009)

\begin{abstract}
Recently, there has been a growing interest in optical imaging of neural activity because the optical neuroimaging has considerable advantages over conventional imaging. Birefringence of the axon has been reported to change during neural activation, but the neurophysiological origin of the change is still unresolved. This study hypothesizes that the birefringence signal is at least partially attributed to the transient cellular volume change associated with nerve excitation. To examine this hypothesis, we investigated how the intensity of cross-polarized light transmitting through the axon would change as the size of the axon changes. For this purpose, a two-dimensional finitedifference time-domain program was developed with the improvement of the total-field/scatteredfield method which reduces numerical noise. The results support our hypothesis in that the computed cross-polarized signals exhibit some agreement with previously-reported birefringence signals.
\end{abstract}

Keywords : Neural activity, Birefringence signal, Neurophysiological origin, Finite-difference timedomain

OCIS codes : (170.0170) Medical optics and biotechnology; (170.3660) Light propagation in tissues; (170.5380) Physiology; (260.1440) Birefringence

\section{INTRODUCTION}

In recent years, the brain has been studied with unprecedented interest in the field of neuroscience and in neural engineering applications as well. Since the brain basically consists of a network of neurons, measurement of dynamic activation in large neural populations is one of the most essential parts in brain studies. It would be ideal if the neural activation could be imaged with a single cell spatial resolution across a large tissue area with high temporal resolution. To date, several techniques have been developed to record neural activity. One of the most widely used methods is the measurement by an electrode located near the neuronal cells in the tissue. The electrode directly measures small fluctuations in the electrical potential resulting from neural activity, which is known as the local field potential. Although this method provides robust recording with high temporal

*Corresponding author: kimsj@snu.ac.kr resolution, it has some limitations. Insertion of the electrodes can damage neural tissues, which is particularly problematic in human studies. In addition, a long-term recording is very hard because inserted electrodes occasionally stop working after a long time due to cellular sheath encapsulation. In studies employing electrical stimulation, the signals recorded with electrodes are often contaminated by stimulus artifacts.

For these reasons, there has been a growing interest in the development of a noninvasive and artifact-free method of recording neural activity. A number of investigators are trying to develop such a technique by using optical methods. Optical recording from an isolated single axon or nerve has been studied mainly because it could serve as a basis for the optical technique. In particular, fast intrinsic (label-free) changes in the birefringence (BIR) and scattering associated with neural activity were reported $[1,2]$. According to their studies, the BIR signal was larger than the scattering one by one order 
of magnitude. Hence, some investigators are using these BIR signals to develop the noninvasive optical technique.

In spite of the usefulness of the BIR signals, neurophysiological origins underlying the signals still remain unresolved. It is certain that a better understanding of the mechanism will improve the BIR signal-based neural recording techniques. The origin is elusive because the time course (waveform) of the BIR signal is quite different from the electrical signal [2]. The distinct waveform suggests that the BIR change is dominantly governed by neurophysiological events other than the membrane potential itself. Although some investigators proposed the hypothesis that the BIR signals could arise from the reorientation of membrane proteins caused by the membrane potential change, it is difficult to explain the difference in the time courses [1,2]. Other investigators suggested that the BIR changes might originate from the cell swelling associated with neural excitation [3].

We hypothesize that the transient cellular volume change (tCVC) during neural activation underlies at least part of the BIR signals. Since the tCVC involves osmosis-driven water flux, we expect that the tCVC would have a time course different from that of the membrane potential. In recent studies, tCVC has been directly measured using the spectral domain optical coherence tomography (SD-OCT), and the results show that the time course of tCVC was very similar to the BIR signals [4]. There still remains one important question in linking the tCVC to the BIR signals, which is how the mechanical tCVC actually produces a change in the optical birefringence. This study is aimed at theoretically investigating the effects of the tCVC on the BIR. For this purpose, we developed a finite-difference time-domain (FDTD) program using recent techniques such as the uniaxial perfectly matched layer (UPML). We also improved our FDTD program by proposing a novel method in applying incident wave sources, which significantly reduced numerical noise stemming from field leakage at the total-field/scattered-field (TF/SF) interface. Using the custom-built FDTD program, this study calculates how the BIR is altered by the tCVC, and compares the computational results to previouslyreported measurements.

\section{COMPUTATIONAL METHOD}

\section{Yee's algorithm}

The FDTD method, introduced by Yee in 1966 [5], provides direct time-domain solutions of Maxwell's differential (curl) equations on spatial grids. This FDTD has been used in a wide range of applications including biomedical photonics $[6,7]$.

Yee's algorithm discretizes Maxwell's two curl equations in space and time, which results in a set of six finitedifference equations for six electromagnetic fields, $E_{x}$,
$E_{y}, E_{z}, H_{x}, H_{y}$ and $H_{z}$ where $E$ is the electrical field and $H$ is the magnetic field. In this study, we reduced the equations to the two-dimensional case, because the structure being modeled here, a neuronal axon is symmetric in its long axis (the z-direction). Hence the six fields were updated in the x-yplane through time-stepping.

\section{Absorbing boundary conditions}

Many problems, including the scattering computation of arbitrary objects, require open regions where the spatial domain under calculation is unbounded. For this reason, the FDTD needs suitable boundary conditions that can simulate extension to infinity. This can be achieved by absorbing boundary conditions (ABCs) that suppress numerical reflections of outgoing waves to an acceptable level.

One of the most efficient ways to realize such ABCs is to locate an absorbing medium at the outer boundary of the domain. The perfectly matched layer (PML), introduced by Berenger, has been widely used for this purpose [8]. Recently, a uniaxial anisotropic medium having both magnetic and electric permittivity tensors was proposed to be utilized as an ABC. In this study, we implemented the $\mathrm{ABC}$ using this uniaxial PML (UPML) formulation $[9,10]$, which resulted in a numerical reflection smaller than $10^{-4}$ of the outgoing field.

\section{Incident wave sources}

To simulate the field propagation in the numerical space, electromagnetic wave excitations should be introduced. One of the most widely-used methods is the total-field/scattered-field ( $\mathrm{TF} / \mathrm{SF}$ ) formulation. It is particularly useful in plane wave sources $[11,12]$. The $\mathrm{TF} / \mathrm{SF}$ technique excites the plane wave source at the $\mathrm{TF} / \mathrm{SF}$ interface by using pre-calculated field values of the incident plane wave. In this process, the conventional method uses a look-up table of the standard plane wave. For arbitrary plane wave sources or during timesteps after the initial excitation, proper interpolations from the standard look-up table are used. Although this method is very powerful and efficient in memory usage, there is a numerical field leakage at the TF/SF interface. In particular, field leakage can be problematic in noisesensitive calculations like those in this. Therefore, we improved the TF/SF method to significantly reduce field leakage.

We built a look-up table of all spatiotemporal values of incident fields for a sufficiently large time-stepping. Every field value at the TF/SF interface was recorded when the incident wave propagated in an empty space full of the background medium. Those field values were used in subsequent simulations conducted with the subject of interest. Although this method is not very efficient in calculation time and memory usage, it suppresses field leakage to almost zero. As shown in Figure 1, after long time-steps, while several percent of the incident field 


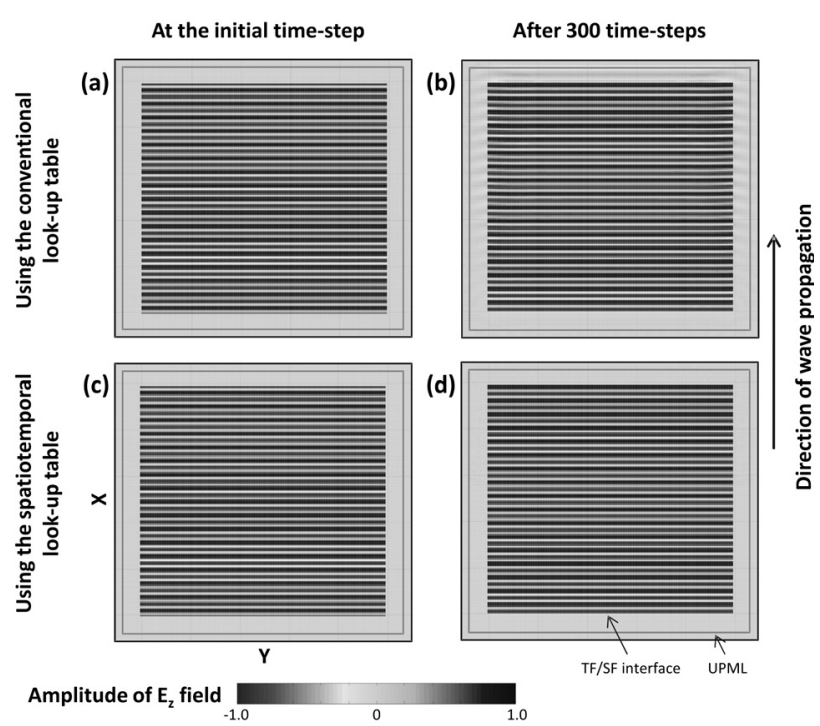

FIG. 1. Performance comparison of the spatiotemporal lookup table and the conventional method. Propagating $\mathrm{E}_{\mathrm{z}}$ fields are displayed as color maps for four cases: (a) at the initial time in the simulation using the conventional look-up table; (b) after 300 time-steps in the same simulation; (c) at the initial time in the simulation using our spatiotemporal look-up table; and (d) after 300 time-steps in the same simulation to (c). At the initial time, there was no field leakage at the TF/SF interface with the conventional method as well as with the spatiotemporal table. However, after 300 time-steps, the field leakage with the conventional method was much larger than with the spatiotemporal table. The total-field zone spans $200 \times 200$ cells, and is surrounded on all sides by a 10-cell scattered-field zone. The horizontal axis represents the y-axis while the vertical one represents the $\mathrm{x}$-axis, the propagating direction of the incident wave source. The gray box indicates the interface between the SF zone and UPML.

leaked from the TF/SF interface in the conventional method, field leakage was smaller than $10^{-10}$ when our spatiotemporal look-up table was used.

\section{Near-to-far-field transformation for cross-polarized scattering pattern}

Since the FDTD method computes field values only in the region around the subject, the obtained values are near-field data. To compare the computation with experimental results observed in the far-field configuration, it is necessary to transform the near-field data to the far-field data. This process can be done by using the phasor-domain Green's theorem [13].

First, time-domain values of near-fields were converted to frequency-domain values by a discrete Fourier transform. Then, the equivalent electric and magnetic current densities $(\vec{J}$ and $\vec{M}$, respectively) can be obtained as

$$
\begin{aligned}
& \vec{J}\left(\overrightarrow{r^{\prime}}\right)=\hat{n}^{\prime} \times \vec{H}\left(\overrightarrow{r^{\prime}}\right) \\
& \vec{M}\left(\overrightarrow{r^{\prime}}\right)=-\hat{n}^{\prime} \times \vec{E}\left(\overrightarrow{r^{\prime}}\right)
\end{aligned}
$$

where $\overrightarrow{r^{\prime}}$ is a point on the closed loop surrounding the structure and $\hat{n}^{\prime}$ is a unit vector normal to the loop at $\overrightarrow{r^{\prime}}$. Using the Green function in two dimensions (the Hankel function), the vector potentials at far-field were computed by integrating near-field current densities [12]:

$$
\begin{aligned}
& \vec{A}(\vec{r})=\frac{\mu_{0}}{\sqrt{8 \pi k}} \frac{e^{-j k r}}{\sqrt{r}} e^{-j(3 \pi / 4)} \int_{\text {loop }} \vec{J}\left(\overrightarrow{r^{\prime}}\right) e^{j k\left(x^{\prime} \cos \phi+y^{\prime} \sin \phi\right)} d l^{\prime}, \\
& \vec{F}(\vec{r})=\frac{\varepsilon_{0}}{\sqrt{8 \pi k}} \frac{e^{-j k r}}{\sqrt{r}} e^{-j(3 \pi / 4)} \int_{\text {loop }} \vec{M}\left(\overrightarrow{r^{\prime}}\right) e^{j k\left(x^{\prime} \cos \phi+y^{\prime} \sin \phi\right)} d l^{\prime}
\end{aligned}
$$

where $\vec{A}$ is the magnetic vector potential, $\vec{F}$ is the electrical vector potential, $k$ is the wave number, $\int_{\text {loop }} d l^{\prime}$ means the line integral along the closed-boundary of near-field zone, $\vec{r}$ is an observation point in the far field, and $\phi$ is the angle between $\vec{r}$ and x-axis. The above formulations assume that the observation point $\vec{r}$ is in the far field away from any points on the closed loop. Based on these vector potentials, far-field electric and magnetic field components were given by [12]:

$$
\begin{aligned}
& E_{x}=-j \omega A_{x}+\frac{j k}{\varepsilon_{o}}\left(F_{z} \sin \phi\right), \\
& E_{y}=-j \omega A_{y}+\frac{j k}{\varepsilon_{o}}\left(-F_{z} \cos \phi\right), \\
& E_{z}=-j \omega A_{z}+\frac{j k}{\varepsilon_{o}}\left(-F_{x} \sin \phi+F_{y} \cos \phi\right), \\
& H_{x}=-j \omega F_{x}-\frac{j k}{\mu_{o}}\left(A_{z} \sin \phi\right), \\
& H_{y}=-j \omega F_{y}+\frac{j k}{\mu_{o}}\left(-A_{z} \cos \phi\right), \\
& H_{z}=-j \omega F_{z}+\frac{j k}{\mu_{o}}\left(-A_{x} \sin \phi+A_{y} \cos \phi\right)
\end{aligned}
$$

where $\omega$ is the frequency of incident wave and $\frac{\partial}{\partial_{Z}}=0$ from the symmetry was used. Then, the far-field scattering pattern $P_{S C}$ can be calculated by

$$
P_{S C}(\vec{r})=\frac{1}{2} \operatorname{Re}\left[\vec{E}(\vec{r}) \times \vec{H}^{*}(\vec{r})\right] .
$$

Additionally, since the BIR signal should be computed in this study, we first calculated the cross-polarized electric field $\left(E_{c p}\right)$ of light transmitting the polarizer that is located at the far-field scattering angle $\left(\alpha+\frac{\pi}{2}\right)$ 


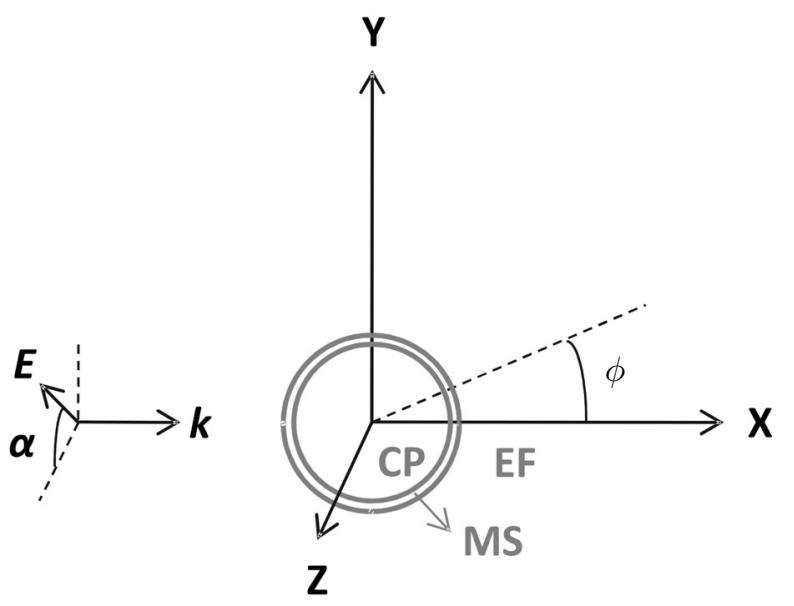

FIG. 2. Schematics of the FDTD simulation. The axon was located along the z-axis. The incident light of electric field $E_{o}$ and polarization angle $\alpha$ was introduced with the wave propagation vector $\mathrm{k}$ parallel to the $\mathrm{x}$-axis. $\phi$ is the angle of far-field scattering. The axon, merged in the extracellular fluid (EF), was modeled by the cylindrical cytoplasm (CP) surrounded by the membrane and sheath (MS).

crossed to the incident polarization $(\alpha)$. Similarly, we calculated the magnetic field $\left(H_{c p}\right)$ corresponding the cross-polarized electric field:

$$
\begin{aligned}
& E_{c p}=E_{x} \cos \left(\alpha+\frac{\pi}{2}\right)-\left(E_{y} \cos \phi-E_{z} \sin \phi\right) \sin \left(\alpha+\frac{\pi}{2}\right), \\
& H_{c p}=H_{x} \cos (\alpha+\pi)-\left(H_{y} \cos \phi-H_{z} \sin \phi\right) \sin (\alpha+\pi)
\end{aligned}
$$

where $\alpha$ is the polarization angle of the incident light relative to the orientation of the long axis of the axon. Then, the far-field cross-polarized scattering pattern for each scattering angle $\phi$ was obtained:

$$
P_{c p}(\phi)=\frac{1}{2} \operatorname{Re}\left(E_{c p} H_{c p}^{*}\right) .
$$

Our FDTD program was verified by comparing the computed scattering pattern of homogeneous cylinders to the analytic solution of Mie theory.

\section{Simulation parameters}

It is well known that the grid spacing $(\Delta)$ and time step $(\Delta t)$ affect the numerical error of FDTD simulation. In two-dimensional cases, the Courant stability factor $S=c \Delta t / \Delta$ should be smaller than $1 / \sqrt{2}$ to achieve numerical stability. The grid sampling density $N_{\lambda}=\lambda_{0} /$ $\Delta$ is directly related to the numerical error. Usually, the numerical phase-velocity error is approximately $1 \%$ when $N_{\lambda}=12$, and $0.1 \%$ when $N_{\lambda}=36$ [14]. In this study, $\mathrm{S}$ was chosen to be $1 / 2$, and $N_{\lambda}$ was 16 for an acceptably small numerical error along with reasonable computation memory.

In the geometric configuration illustrated in Figure 2,
TABLE 1. Variable parameters in our simulations.

\begin{tabular}{c|c|c|c|c}
\hline \hline Symbol & Parameter & Values & Unit & \#Cases \\
\hline $\mathrm{D}$ & $\begin{array}{c}\text { Axon } \\
\text { diameter }\end{array}$ & $\begin{array}{c}48,48.5,49,49.5,50.5,51,51.5, \\
50,5 \mathrm{~m}\end{array}$ & 9 \\
\hline \multirow{2}{*}{$\alpha$} & $\begin{array}{c}\text { Incident } \\
\text { polarization } \\
\text { angle }\end{array}$ & 0,45, and 90 & $\operatorname{deg}$ & 3 \\
\hline \multicolumn{4}{|c}{ Total number of cases } & 27 \\
\hline
\end{tabular}

we first recorded near-field data during one period after sufficiently large time-stepping, and computed the farfield cross-polarized scattering pattern for various axon diameters and polarization angles. The axon was modeled by a concentric cylinder where the inner cylinder represented the cytoplasm and the outer cylindrical shell was the membrane and sheath surrounding the cytoplasm. The indices of refraction for the extracellular fluid, cytoplasm and membrane were taken from the literature in the values of $1.35,1.37$ and 1.48 , respectively [15-17]. The initial diameter of the cytoplasm was $50 \mu \mathrm{m}$, and the thickness of the boundary was fixed to $1 \mu \mathrm{m}$. The diameter of the cytoplasm varied by $\pm 1, \pm 2, \pm 3$ and $\pm 4 \%$ from its initial value. For each diameter, the crosspolarized scattering patterns $P_{c p}(\phi)$ were computed for the incident polarization angle $\alpha$ of 0,45 and 90 degrees. Simulation variables are listed in Table 1 . The wavelength of the light in this study was $800 \mathrm{~nm}$.

\section{RESULTS AND DISCUSSION}

\section{FDTD computation for a single case}

In the FDTD computation with the modeled axon, electromagnetic fields were updated as time-stepping progressed, one example of which is displayed in Figure 3. Since the axon consisted of the cytoplasm and membrane, the waves propagated in complicated manners. Based on these near-field data, the far-field values were computed and used to calculate the scattering pattern $P_{s c}$ and the cross-polarized scattering $P_{c p}$. Figure 4 shows these patterns in the case of $\mathrm{TE}_{z}$ incident wave $(\alpha=90$ $\mathrm{deg}$ ), for example. Although the cross-polarized scattering intensities were much smaller than the whole scattering intensities, they are normalized by their own maximum values for angular pattern comparison.

\section{Computation results from all cases}

We obtained the cross-polarized scattering patterns for a total of 27 cases, a combination of 9 axon diameters and 3 polarization angles, as listed in Table 1. From each pattern, the cross-polarized intensity $I_{c p}$ was calculated by integrating the scattering intensities for $\phi<$ $\pi / 12$, which is a typical angle of the collimating lens 


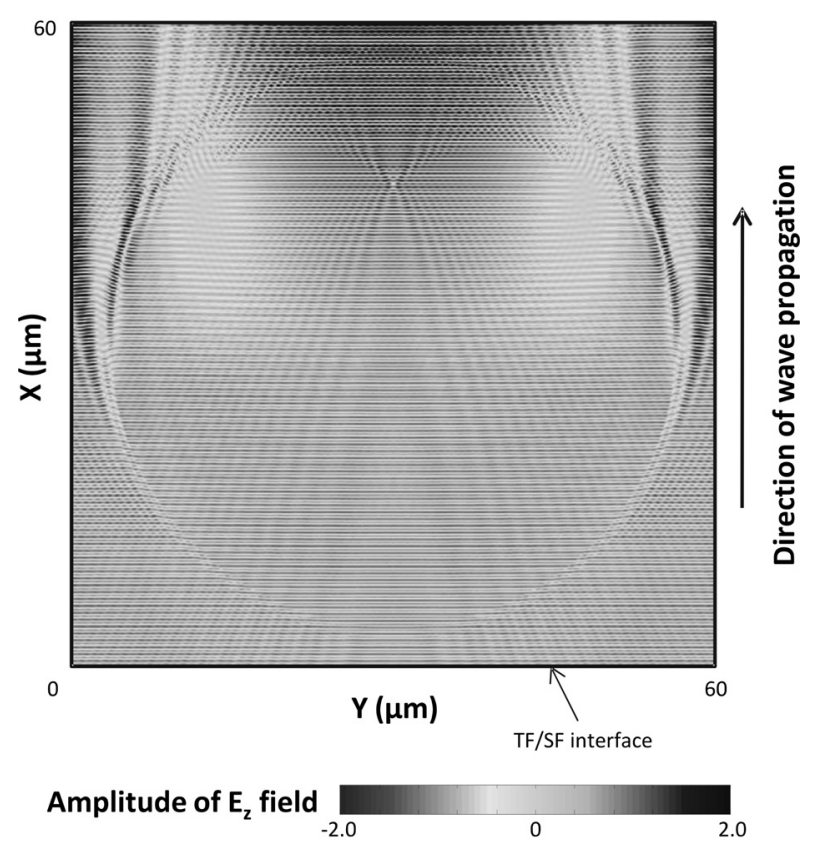

FIG. 3. Example snapshot of the $E_{z}$ field at time-steps 4270 in the case of $50 \mu \mathrm{m}$ axon diameter and $\mathrm{TM}_{\mathrm{z}}$ incident wave source. The $E_{z}$ field is displayed only in the total-field zone.

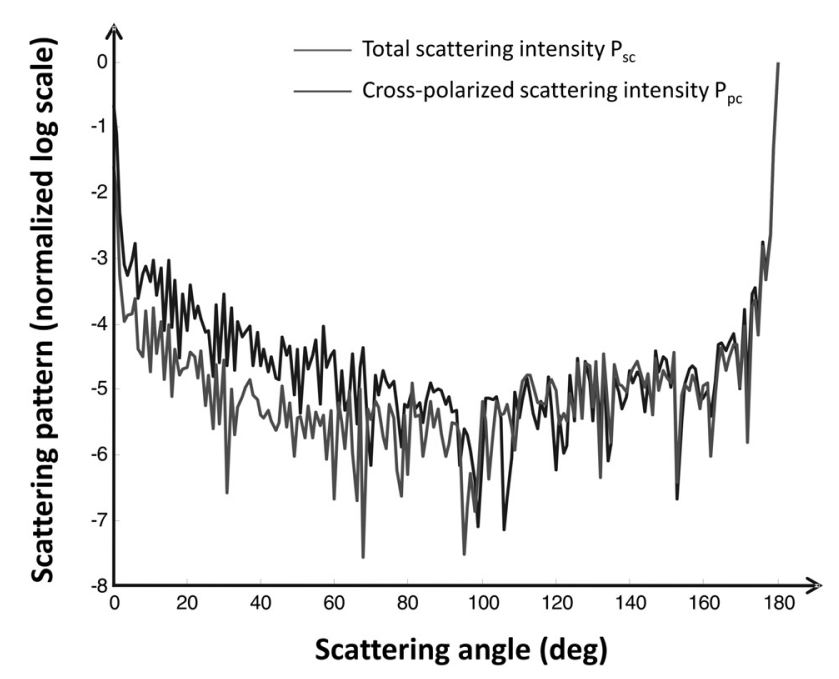

FIG. 4. Scattering patterns in the case of 50 ' $\mathrm{m}$ axon diameter and $\mathrm{TE}_{\mathrm{z}}$ incident wave source. The blue line shows the scattering pattern $P_{s c}$ while the red line shows the cross-polarized scattering pattern $P_{c p}$. Intensities are normalized by the maximum of each pattern, and displayed in log scale. 4270 in the case of $50 \mu \mathrm{m}$ axon diameter and $\mathrm{TM}_{\mathrm{z}}$ incident wave source. The $E_{z}$ field is displayed only in the total-field zone.

(variation of this angle in a reasonable range produced little change in our results). Relative changes in the cross-polarized intensity $\left(\Delta I_{c p} / I_{c p}\right)$ caused by axon size variation $(\Delta \mathrm{D} / \mathrm{D})$ are illustrated as circles in Figure 5.

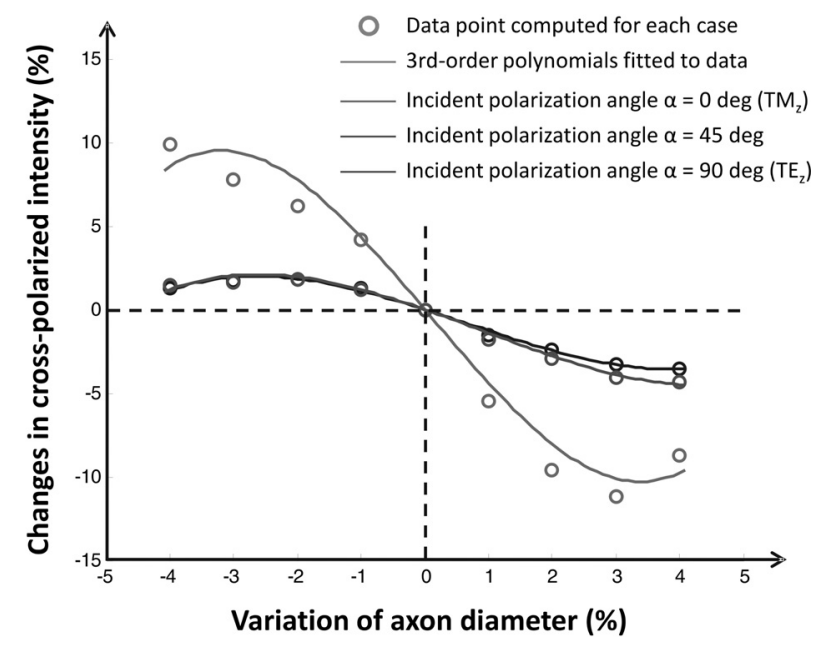

FIG. 5. Changes in the cross-polarized intensity associated with axon size variation. The blue circles are computed intensity changes when the $\mathrm{TM}_{\mathrm{z}}(\alpha=0 \mathrm{deg})$ wave source was introduced. The magenta and red circles are for the case of $a=45$ and $90 \mathrm{deg}\left(\mathrm{TE}_{\mathrm{z}}\right)$, respectively. Solid color lines represent the third-degree polynomials fitted to the result of the same color.

TABLE 2. Fitting coefficients for various polarizations of the incident wave source. $\alpha$ is the polarization angle relative to the long axis of the axon. $C_{1}, C_{2}$ and $C_{3}$ are coefficients of the third-degree polynomials (Equation 6) fitted to the cross-polarized intensity results from the FDTD computation. See Equation 7 and related description for $Z_{\text {sens }}$.

\begin{tabular}{c|c|c|c}
\hline \hline$\alpha(\mathrm{deg})$ & $C_{1}$ & $C_{2}$ & $C_{3} \approx Z_{\text {sens }}$ \\
\hline 0 & 398.4 & -7.570 & -1.229 \\
\hline 45 & 1379 & -3.702 & -4.500 \\
\hline 90 & 395.9 & -10.32 & -1.342 \\
\hline
\end{tabular}

\section{Response direction of BIR signals}

According to our computation results, the crosspolarized intensity decreases when the axon size is enlarged. As described above, recent studies demonstrated using the SD-OCT that the axon diameter increased during neural activation [4]. Combined with this experimental result, our results suggest that the BIR intensity will be reduced during nerve excitation. Remarkably, this agrees with previously-observed BIR signals $[1,2]$.

\section{BIR signal amplitudes depending on the incident polarization}

Our results also indicate that the cross-polarized signal with 45-deg polarized light is larger than those with other polarizations. This tendency is again in agreement with the measured BIR signals [1,2]. In addition, we fitted results to the following 3rd-degree polynomial: 


$$
\frac{\Delta I_{c p}}{I_{c p}}=C_{1}\left(\frac{\Delta D}{D}\right)^{3}+C_{2}\left(\frac{\Delta D}{D}\right)^{2}+C_{3}\left(\frac{\Delta D}{D}\right)
$$

where $C_{1}, C_{2}$, and $C_{3}$ are fitting coefficients. The above polynomial is assumed to cross the origin of the coordinate axes because the relative change $\Delta I_{c p} / I_{c p}$ is defined by variation from a baseline that is the intensity when the axon diameter is the initial value $(\Delta D=0)$. Fitting results are illustrated as solid lines in Figure 5, and fitting coefficients are listed in Table 2. Since the amplitude of the tCVC in measurement is quite small $\left(\sim 10^{-5}\right.$ of its original size), the sensitivity of the cross-polarized signal $Z_{\text {sens }}$ can be defined by

$$
\left.Z_{\text {sens }} \equiv \frac{\Delta I_{c p} / I_{c p}}{\Delta D / D}\right|_{\frac{\Delta D}{D} \rightarrow 0} \cong C_{3}
$$

As can be seen in Table 2, the sensitivity with 45-deg polarization is about 3.5 times larger than those with 0 and 90-deg polarizations. This computation result suggests that the BIR signal in an experiment would be more readily detected when the light of 45-deg polarization is used rather than $\mathrm{TM}_{\mathrm{z}}$ or $\mathrm{TE}_{\mathrm{z}}$ incident light. This tendency is also consistent with the measurement results $[1,2]$.

\section{Discussion}

The cross-polarized signal sensitivity is larger than one when the 45-deg polarization light is used. This means that the BIR measurement will amplify the variation in the axon size during nerve excitation. If we accepted the hypothesis that the polarization-free scattering is directly related to the tCVC and so the scattering signal is similar in amplitude to the tCVC, our result of $Z_{\text {sens }}$ $>1$ could explain why the BIR signal was significantly larger than the scattering signal in the experiments.

In addition, the time course of the tCVC from the measurement [4] was similar to the BIR signals [3]. Accordingly, together with this coincidence in the experiments, our results support our hypothesis that the BIR signal at least partially originates from the tCVC.

As another issue to be discussed, this study did not consider the intrinsic BIR of the axon (iBIR) in our simulations. An axon can have its iBIR, because the membrane of the axon is electrically polarized and resultant equivalent membrane dipoles are cylindrically distributed. Further, the iBIR will affect the resting pattern of cross-polarized scattering $\left(P_{c p}\right)$. Nevertheless, we did not consider the iBIR for two reasons. First, as briefly described in the Introduction, a change in the iBIR will have a time course following the membrane potential. Since previous reports showed that the BIR signal has the time course different from the electrical signal [3], we initially excluded the hypothesis of membrane dipole reorientation. Second, although the iBIR can affect the resting $I_{c p}$, it would not play a critical role in the relative change of $I_{c p}\left(\Delta I_{c p} / I_{c p}\right)$. Since the membrane dipole-oriented iBIR is localized into the tiny space of membrane, the contribution of iBIR to the resting $I_{c p}$ will be not drastically different between the $50-\mu \mathrm{m}$ axon and $51-\mu \mathrm{m}$ axon, for example. It implies that the iBIR will affect the $\Delta$ $I_{c p} / I_{c p}$ less compared to the contribution to the resting $I_{c p}$. For these reasons, the iBIR was not considered in this study, which focused on the relative change that showed a time course different from the electrical signal.

\section{CONCLUSION}

In this study, we investigated whether the tCVC can feasibly be an origin underlying the BIR signals. For this purpose, we first developed a two-dimensional FDTD program, in which the TF/SF method was improved to reduce the numerical noise due to field leakage. Using this FDTD program, cross-polarized intensities were computed for various axon diameters and incident polarizations. Our results show that the cross-polarized intensity can change when the axon size is varied, with three characteristics: (1) the intensity decreases as the axon size is enlarged; (2) the relative change in the intensity is larger when the 45 deg-polarization light is used; and (3) the intensity change amplifies the size variation. Remarkably, all of these characteristics are in good agreement with the tendencies of the measured BIR signals. Considering, additionally, that the temporal dynamics of the tCVC was similar to the BIR signals, we can conclude that the tCVC is one of the possible neurophysiological origins underlying the BIR signals.

Future work will include the mathematical modeling of the tCVC. Since the conventional Hodgkin-Huxley model cannot describe a volume change during neural activation, we are currently developing a novel neuron model which includes the cellular volume and ion concentrations as variables. In addition, more realistic FDTD simulation can be performed with multiple axons and other cellular compositions such as the fascicular sheath and peri-fascicular spaces, which will be possible when large computer memory is available.

\section{ACKNOWLEDGMENT}

This work was supported by the ERC program of MEST/KOSEF (grant \#R11-2000-075-01001-0), and the grant from the Industrial technology development program (10031270) of the Ministry of Knowledge Economy (MKE) of Korea. 


\section{REFERENCES}

1. L. B. Cohen, R. D. Keynes, and B. Hille, "Light scattering and birefringence changes during nerve activity," Nature 218, 438-441 (1968).

2. I. Tasaki, A. Watanabe, R. Sandlin, and L. Carnay, "Changes in fluorescence, turbidity, and birefringence associated with nerve excitation,” Proc. Natl. Acad. Sci. USA. 61, 883-888 (1968).

3. K. M. Carter, J. S. George, and D. M. Rector, "Simultaneous birefringence and scattered light measurements reveal anatomical features in isolated crustacean nerve," J. Neurosci. Meth. 135, 9-16 (2004).

4. T. Akkin, C. Joo, and J. F. Boer, "Depth-resolved measurement of transient structural changes during action potential propagation,” Biophys. J. 93, 1347-1353 (2007).

5. K. S. Yee, "Numerical solution of initial boundary value problems involving Maxwell's equations in isotropic media,” IEEE Trans. Antennas Propagat. 14, 302-307 (1966).

6. R. Drezek, A. Dunn, and R. Richards-Kortum, "Light scattering from cells: finite-difference time-domain simulations and goniometric measurements," Appl. Opt. 38, 3651-3661 (1999).

7. R. Drezek, A. Dunn, and R. Richards-Kortum, "A pulsed finite-difference time-domain (FDTD) method for calculating light scattering from biological cells over broad wavelength ranges," Opt. Exp. 6, 147-157 (2000).

8. J. P. Berenger, "A perfectly matched layer for the absorption of electromagnetic waves," J. Computational Physics 114, 185-200 (1994).
9. S. D. Gedney, "An anisotropic PML absorbing media for FDTD simulation of fields in lossy dispersive media," Electromagnetics 16, 399-415 (1996).

10. S. D. Gedney, "An anisotropic perfectly matched layer absorbing media for the truncation of FDTD lattices," IEEE Trans. Antennas Propagat. 44, 1630-1639 (1996).

11. D. E. Merewether, R. Fisher, and F. W. Smith, "On implementing a numeric Huygen's source scheme in a finite difference program to illuminate scattering bodies," IEEE Trans. Nuclear Science 27, 1829-1833 (1980).

12. K. R. Umashankar and A. Taflove, "A novel method to analyze electromagnetic scattering of complex objects," IEEE Trans. Electromagn. Compat. 24, 397-405 (1982).

13. R. Luebbers, S. Kunk, M. Schneider, and F. Hunsberger, "A finite-difference time-domain near zone to far zone transformation," IEEE Trans. Antennas Propagat. 39, 429-433 (1991).

14. A. Taflove and M. Brodwin, "Numerical solution of steadystate electromagnetic scattering problems using the timedependent Maxwell's equations," IEEE Trans. Microwave Theory Tech. 23, 623-630 (1975).

15. J. Maier, S. Walker, S. Fantini, M. Franceschini, and E. Gratton, "Possible correlation between blood glucose concentration and the reduced scattering coefficient of tissues in the near infrared," Opt. Lett. 19, 2062-2064 (1994).

16. A. Brunsting and P. Mullaney, "Differential light scattering from spherical mammalian cells,” Biophys. J. 14, 439-453 (1974).

17. J. Beuthan, O. Minet, J. Helfmann, M. Herrig, and G. Muller, "The spatial variation of the refractive index in biological cells," Phys. Med. Biol. 41, 369-382 (1996). 